\title{
Uso de las Tecnologías de la Información y la Comunicación en Educación Matemática. Una experiencia en las titulaciones de ingeniería de la Universidad de Málaga.
}

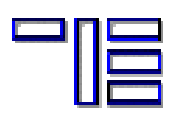

\author{
José Luis Galán García \\ jl_galan@uma.es \\ José Luis González Marí \\ gmari luma.es \\ Yolanda Padilla Domínguez \\ ypadilla@ctima.uma.es \\ Pedro Rodríguez Cielos \\ prodriguez@uma.es \\ Universidad de Málaga
}

1. Las Tecnologías de la Información y la Comunicación (TIC) en la Enseñanza de las Matemáticas.

La Enseñanza de las Matemáticas debe tener en cuenta que es necesario poner al alumno en condiciones que favorezcan su integración social. Esto nos lleva a considerar las características de la sociedad actual y en particular la que se refiere a los avances tecnológicos, que se suceden a tal ritmo que es difícil prever cuáles van a ser los conocimientos necesarios para el hombre del mañana. En esta adaptación a los cambios sociales es esencial el uso de las TIC.

Por otra parte, como los ordenadores y programas de cálculo constituyen herramientas de uso habitual en la actividad profesional de los ingenieros, está bastante extendida hoy día su utilización y en particular la de los Computer Algebra Systems (CAS) en las clases de las asignaturas de Matemáticas en las carreras técnicas (Morphett, 1997). Además, la utilización del ordenador viene a simplificar enormemente la realización de ejercicios y las aplicaciones usuales de la materia a los problemas propios de la Ingeniería, lo que hace que sea especialmente indicado su uso en el periodo de formación.

Sin embargo, el uso de los CAS se reduce, en la mayoría de los casos, a utilizar el ordenador como una calculadora potente de altas prestaciones, lo que representa claramente una infrautilización de los recursos, por lo que se hace necesario un cambio de punto de vista para optimizar las oportunidades que ofrecen las TIC y tratar de fomentar la creatividad matemática de los alumnos (Ortega, 2002; Galán y otros, 2002a; Galán y otros, 2002b).

En los últimos años se está produciendo un fuerte movimiento dentro del colectivo de profesores que utilizan CAS en la enseñanza de las Matemáticas con el fin de cambiar los usos didácticos tradicionales de estas herramientas. Se deben modificar dichos usos para maximizar las oportunidades que ofrecen estas tecnologías (Garcia y otros, 2002), orientando su aplicación, por ejemplo, en el sentido de incidir positivamente en el aprendizaje (Dubinsky y Noss, 1996), aumentar considerablemente la posibilidad de experimentación (Hoya y otros, 2002) y permitir que el alumno construya su conocimiento matemático bajo la orientación del docente (Nava, 1998). Es en esta dirección en la que venimos desarrollando nuestros trabajos.

Pero para ello es necesario que se elaboren actividades apropiadas. Por ejemplo, se deben proponer 
problemas de muy difícil resolución sin el uso de CAS, puesto que no se pueden resolver los mismos problemas que se resolvían cuando no existían los CAS (Abboud, 2002; Westermann, 2000) y se deben hacer unos ejercicios y problemas mucho más realistas aprovechando las posibilidades de los ordenadores (Monagan, 1994). Por otro lado, puesto que a veces los CAS no presentan los resultados en el modo usual o esperado, se pueden utilizar dichos resultados inesperados para reforzar el aprendizaje de los conceptos y fomentar el espíritu crítico de los alumnos (Alonso y otros, 2001). Incluso se pueden aprovechar los "errores" que cometen los CAS para ayudar en el proceso de enseñanza-aprendizaje (Bovio, 2002).

En otro orden de cosas, estamos de acuerdo con los profesores que afirman que el uso de CAS en las clases de Matemáticas no ha alcanzado todavía su grado óptimo (Neuper, 2001). De hecho, los CAS más usuales son blackbox (muestran el resultado en un paso sin enseñar cómo se llega a él), mientras que para poder sacar todo el provecho a los CAS, éstos deberían ser whitebox, es decir, mostrar los desarrollos intermedios (Strickland, 1999). En menor medida, no por ser menos importante sino por su novedad, está la idea de utilizar la programación con lenguajes informáticos en las clases de Matemáticas (Dubinsky, 1998). Así, cuando los alumnos programan deben leer, construir y depurar estrategias, modificar programas que ya están desarrollados y, por último, resolver los problemas con dichos programas. Esto les convierte en protagonistas de su aprendizaje (Hector, 2002). Además, el uso de la programación permite encontrar tareas adecuadas que se corresponden directamente con las construcciones mentales de los conceptos matemáticos (Dubinsky, 1994). Como ejemplo se puede citar el lenguaje de programación Isetl, utilizado por el profesor Dubinsky para enseñar Matemáticas a alumnos universitarios por tener una sintaxis que favorece la asimilación de los contenidos matemáticos (Dubinsky, 1995).

Por último, una buena idea es complementar la programación con los CAS mediante la realización de comandos o funciones específicas (Majewski, 2000), lo que permite aumentar considerablemente las librerías de funciones predeterminadas que contienen. Más en concreto, compartimos el enfoque del profesor Böhm, que muestra las posibilidades que ofrece el hecho de combinar la potencia de un CAS (en este caso Derive) con la flexibilidad de un lenguaje de programación (Böhm, 2002). El enfoque que creemos más adecuado es, por tanto, el de utilizar la programación con los CAS para que sean los propios alumnos los que creen las funciones específicas necesarias que les permitan resolver los problemas propios de las materias correspondientes.

\section{Nuestra experiencia.}

Pasamos a continuación a describir la experiencia que llevamos realizando durante los últimos años y que se ha desarrollado en dos asignaturas de la titulación de Ingeniería Técnica de Telecomunicación de la Universidad de Málaga. En concreto, Análisis Vectorial y Ecuaciones Diferenciales, impartida en el segundo cuatrimestre del primer curso con seis grupos de alumnos (unos 600) y Ampliación de Matemáticas, impartida en el primer cuatrimestre del segundo curso con tres grupos de alumnos (unos 300).

Hace unos años nos planteamos desarrollar un estudio sistemático específico de innovación curricular centrado en el proceso real de enseñanza y aprendizaje de dichas asignaturas. En dicho estudio modificamos la metodología didáctica usual mediante la sustitución de una parte de las clases tradicionales de pizarra por clases en el laboratorio de informática utilizando la programación como recurso didáctico y, en particular, la realización de comandos con el programa Derive (Camacho y Depool, 2002).

En consecuencia, los aspectos fundamentales que nos planteamos se pueden resumir en los siguientes interrogantes:

1. ¿Es posible mejorar el proceso didáctico ordinario y sus resultados en las asignaturas de Matemáticas de los estudios de Ingeniería mediante la introducción de la creación de comandos con Derive por parte de los alumnos? 
2. ¿En qué aspectos se consigue la mejora, cuál es el tamaño de los efectos principales y cuáles y cómo son los efectos secundarios?

3. ¿Cómo se puede responder a los interrogantes 1 y 2 sin alterar el proceso ordinario ni afectar negativamente al mismo o a sus resultados?

4. ¿Cuáles son las dificultades y limitaciones que pueden aparecer?

El origen del problema no tiene una fecha puntual ni una situación singular concreta. Antes bien, se sitúa en las experiencias desarrolladas en los últimos diez años. Nos referimos, básicamente, a la realización continuada de prácticas en el laboratorio de informática para algunas de las asignaturas en las que el Departamento de Matemática Aplicada de la Universidad de Málaga tiene asignada docencia, como son las asignaturas de Matemáticas de las titulaciones Ingeniería Técnica Industrial e Ingeniería Técnica de Telecomunicación.

A lo largo de las numerosas experiencias desarrolladas durante la primera parte del periodo mencionado se pudo comprobar, entre otras cosas, la manifiesta infrautilización de que era objeto el ordenador en el aula de Matemáticas, lo que llevó a introducir, en los últimos cuatro años, nuevas actividades orientadas a aprovechar mejor las posibilidades del ordenador y de los programas y a disminuir la utilización exclusiva de los CAS como máquinas de cálculo y para la resolución mecánica de ejercicios. La orientación de las nuevas actividades tenía que ver, principalmente, con la participación activa del alumno en el proceso de enseñanza-aprendizaje y con una mayor atención a la construcción del conocimiento matemático bajo la orientación del docente.

Como consecuencia de la experiencia acumulada en el sentido indicado, se llegó al convencimiento de que podría ser necesario e importante introducir una metodología didáctica basada en la construcción (programación) de comandos con Derive para la mayoría de los contenidos de las asignaturas involucradas en dichas experiencias.

\section{Objetivos de la experiencia.}

El fin general que perseguimos es el de contribuir a la comprensión de la realidad de los procesos y fenómenos de la Educación Matemática en los estudios de Ingeniería, que concebimos dinámica, múltiple, holística y divergente, para poder intervenir sobre ella en orden a su optimización.

Así, para la consecución de esta finalidad general, nos propusimos alcanzar los siguientes objetivos:

- Comprobar los efectos positivos del desarrollo de una metodología didáctica mixta que incluye la realización de comandos con Derive sobre el aprendizaje de las materias de las asignaturas de Matemáticas de la titulación Ingeniería Técnica de Telecomunicación.

- Comprobar la adecuación de la modificación curricular y su compatibilidad con las condiciones usuales del desarrollo de las asignaturas así como con las orientaciones oficiales al respecto.

- Constatar la incidencia positiva del tratamiento didáctico mencionado sobre los diferentes factores del proceso didáctico, sobre las actitudes, los conocimientos, la participación, las destrezas profesionales y el rendimiento de los alumnos.

- Indagar sobre las posibles incidencias negativas que pueda ocasionar la realización de comandos con Derive sobre el aprendizaje de la materia.

- Fomentar la participación del alumnado en la elaboración y desarrollo de las distintas actividades que se proponen, concretamente con la realización de parte de los comandos que se utilizarán para resolver los ejercicios de las asignaturas.

\section{Descripción de la experiencia: metodología, material y métodos.}

Para alcanzar los objetivos enunciados en el apartado anterior, se utilizó una metodología que consistió en la elaboración y realización de prácticas con ordenador en las asignaturas mencionadas y que incluyeron como aspecto innovador la creación (programación) de comandos con el programa 
Derive por parte de los alumnos.

Con este tipo de prácticas, en las que el alumno no sólo resuelve problemas sino que construye los comandos para resolverlos, se pretendía que el uso del ordenador no sólo se viera reducido a su aplicación más clásica y usual de realizar los cálculos a modo de calculadora potente, sino que también se hiciera uso del ordenador como herramienta para fomentar la creatividad matemática de los alumnos.

Así, por ejemplo, para la elaboración de un comando que determine si una forma diferencial es exacta, el alumno debe saber cual es la condición para que una forma diferencial sea exacta, mientras que para la elaboración de los comandos para resolver integrales triples, debe saber que para dicha resolución es necesario considerar: la función a integrar, el sistema de coordenadas elegido para resolverlas y las tres variables de integración con sus correspondientes límites de integración. Además, como el orden de integración influye, necesita tenerlo en cuenta a la hora de la elaboración de los comandos. Posteriormente, le será mucho más sencillo aplicar estos comandos para resolver algún ejemplo concreto puesto que él mismo ha sido quien los ha elaborado.

La elección del programa a utilizar es uno de los puntos más importantes en este proceso. Entre la gran cantidad de software matemático disponible hoy en día se eligió el programa Derive por varios motivos, entre los que destacamos los siguientes:

- Las experiencias acumuladas en tal sentido así como los resultados que se habían venido produciendo en los estudios exploratorios realizados revelaban que el programa Derive es una herramienta informática de fácil manejo y útil para su integración en las clases de Matemáticas de Ingeniería.

- Debido a lo expuesto en el punto anterior, el alumno es capaz de empezar a resolver problemas utilizando el programa en un espacio corto de tiempo, puesto que las funciones y operaciones básicas se encuentran disponibles en diversos menús.

- Había indicios razonables de que la realización de comandos con Derive facilita el aprendizaje y mejora la motivación del alumno.

- Los alumnos a los que estaba dirigida esta experiencia iban a utilizar el programa Derive en otras asignaturas de Matemáticas de la titulación.

Siguiendo el diseño previsto, se elaboraron y desarrollaron tres prácticas para la asignatura Análisis Vectorial y Ecuaciones Diferenciales y dos para la asignatura Ampliación de Matemáticas. Las prácticas desarrolladas tuvieron una estructura común basada en los tres bloques que pasamos a detallar a continuación:

- En el primer bloque se señalan los aspectos teórico-prácticos que se desean desarrollar en la práctica, indicando las funciones propias de Derive y los comandos creados para resolver los problemas que se presentan.

- El segundo bloque consta de ejemplos de aplicación de los conceptos señalados anteriormente y que se resuelven en el transcurso de la práctica. Dentro de estos ejemplos los alumnos elaboran algunos de los comandos con los que resuelven los problemas.

- El tercer bloque se dedica a la realización por parte del alumno de una serie de problemas propuestos, con el fin de que se afiancen los conocimientos adquiridos a lo largo de la práctica. Entre estos ejercicios se incluyen la elaboración de algunos comandos adicionales.

Las dos horas de duración de cada práctica se distribuyó en una hora y media para los dos primeros bloques y media hora para el tercer bloque.

Algunas características adicionales destacables de la realización de estas prácticas se pueden resumir en los siguientes puntos: 
- Las prácticas se realizaron en un laboratorio de forma que los alumnos pudieran utilizar los ordenadores individualmente.

- Las prácticas se desarrollaron de forma guiada, es decir, mediante explicaciones del profesor con la ayuda de un proyector, con idea de realizar los comentarios adecuados para una mejor asimilación de los contenidos presentados.

- Es importante señalar que el desarrollo de las prácticas no sólo se redujo a la realización de los ejemplos de aplicación, sino que se aprovechó cada ejemplo para recordarle al alumno los aspectos teórico-prácticos vistos en "las clases de pizarra", con lo que estas prácticas sirvieron también de repaso de la asignatura.

Por otra parte y a modo de resumen, podemos agrupar el trabajo realizado en las siguientes tres fases:

- La primera fase consistió en la elaboración de las prácticas que se iban a desarrollar posteriormente.

- La segunda constó del desarrollo de las prácticas en cada una de las asignaturas antes comentadas. Recordemos que los alumnos completaron la elaboración de dichas prácticas con la realización (programación) de algunos de los comandos que se iban a utilizar posteriormente.

· En la tercera fase se procedió a la evaluación de las prácticas desarrolladas.

Paralelamente al desarrollo de estas prácticas y dentro de una línea de investigación en Educación Matemática denominada Investigación para la innovación curricular en la acción en el aula de Matemáticas se han realizado tres investigaciones (Galán, 2003; Padilla, 2003; Rodríguez, 2004) que forman parte de un proyecto más amplio de indagación sistemática en varias especialidades y grupos, sobre temas relacionados de diversas asignaturas y carreras de Ingeniería y con la participación efectiva de varios profesores responsables de la docencia correspondiente. Así, se han desarrollado estudios sobre las materias Integrales de Línea, Integrales Múltiples y Derivación e Integración de Funciones de Variable Compleja en los que se analiza la comparación de efectos de una metodología didáctica modificada (que incluye la realización de comandos con Derive) y de la metodología didáctica usual o tradicional.

Por último, señalar que nuestras experiencias se encauzaron bajo los siguientes proyectos, en los que se proponía la elaboración de comandos con Derive como aspecto fundamental:

- El Proyecto de Enseñanza Virtual El ordenador en las asignaturas de Matemáticas de las carreras técnicas: mucho más que una calculadora potente (curso 01/02. Universidad de Málaga).

- El Proyecto de Innovación Educativa Matemáticas para la Ingeniería. El ordenador como herramienta de creatividad matemática (curso 02/03. Universidad de Málaga).

- El Proyecto de Innovación Educativa Elaboración de comandos con Derive. Una experiencia en las titulaciones de Ingeniería Técnica de Telecomunicación (curso 03/04. Universidad de Málaga).

- El Proyecto de Innovación Educativa El uso de la programación como recurso didáctico en la enseñanza de las Matemáticas de las carreras técnicas (curso 04/05. Universidad de Málaga).

- El Proyecto de Innovación Educativa Evaluación en las asignaturas de Matemáticas de las carreras técnicas: ¿por qué reducirla a un único examen final? (curso 04/05. Universidad de Málaga).

- El Proyecto Andaluz de Formación del Profesorado Universitario Nuevos usos de los programas de cálculo simbólico en la enseñanza de las Matemáticas en las titulaciones de Ingeniería (curso 04/05. Unidad de Calidad de las Universidades Andaluzas, código UMA N 016).

\section{Ejemplo de elaboración de comandos.}

En este epígrafe vamos a ver por medio de un ejemplo como los alumnos elaboran los comandos. Para 
ello, centrémonos en la creación de los comandos DOBLE y DOBLEPOLAR para calcular integrales dobles en coordenadas cartesianas y polares respectivamente. Es importante destacar que los comandos son desarrollados paso por paso con el fin de minimizar los posibles errores en su elaboración.

\section{Comando DOBLE}

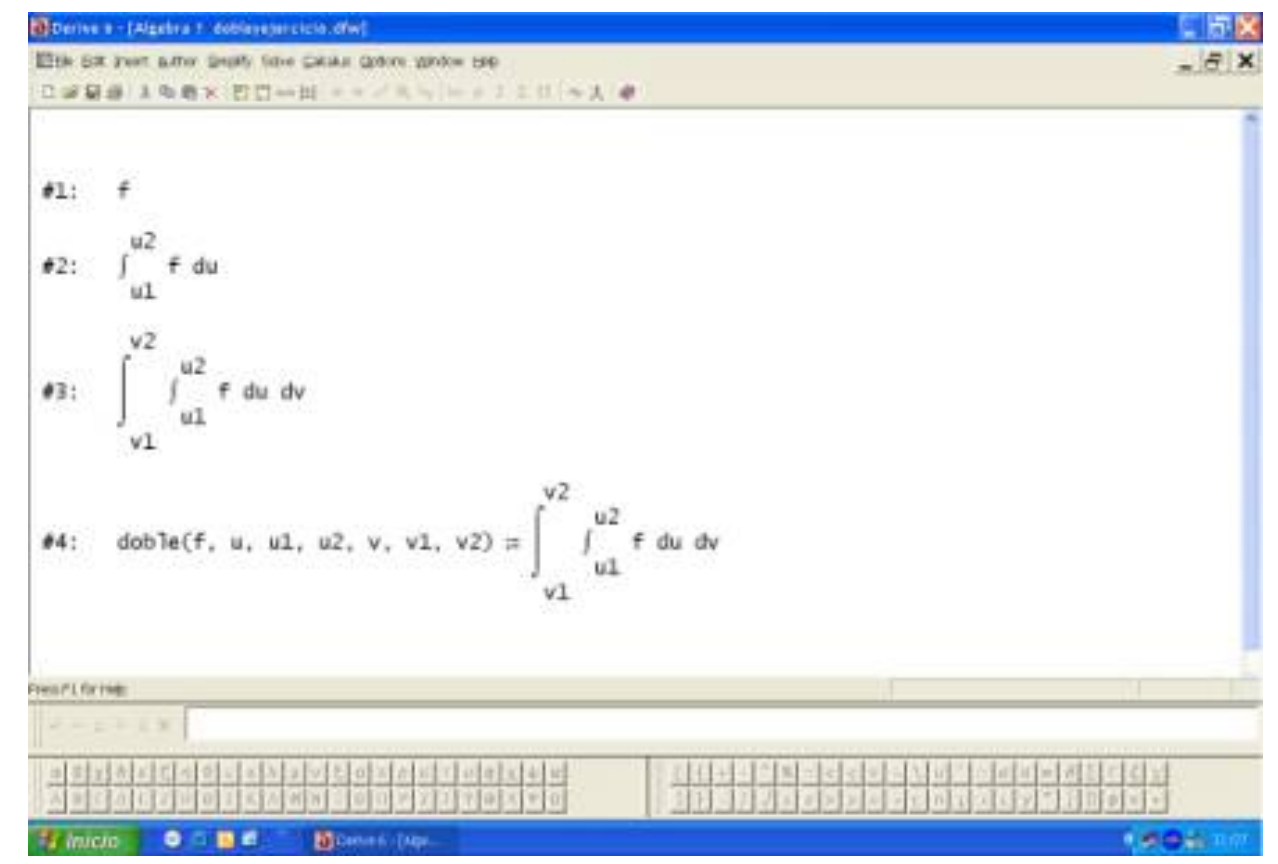

Ilustración 1. Elaboración del comando DOBLE [1]

Una vez que el comando está elaborado, el siguiente paso es resolver un ejercicio de comprobación. En la siguiente figura se muestra la resolución del ejercicio correspondiente al comando DOBLE. Destacar que en este ejercicio también se usan las posibilidades gráficas de Derive.

- Ejercicio de comprobación: Calcular $\iint_{R} x y d x d y$ donde $R$ es la región limitada por $x=0, x=2, y=0$ e $y=x+1$.

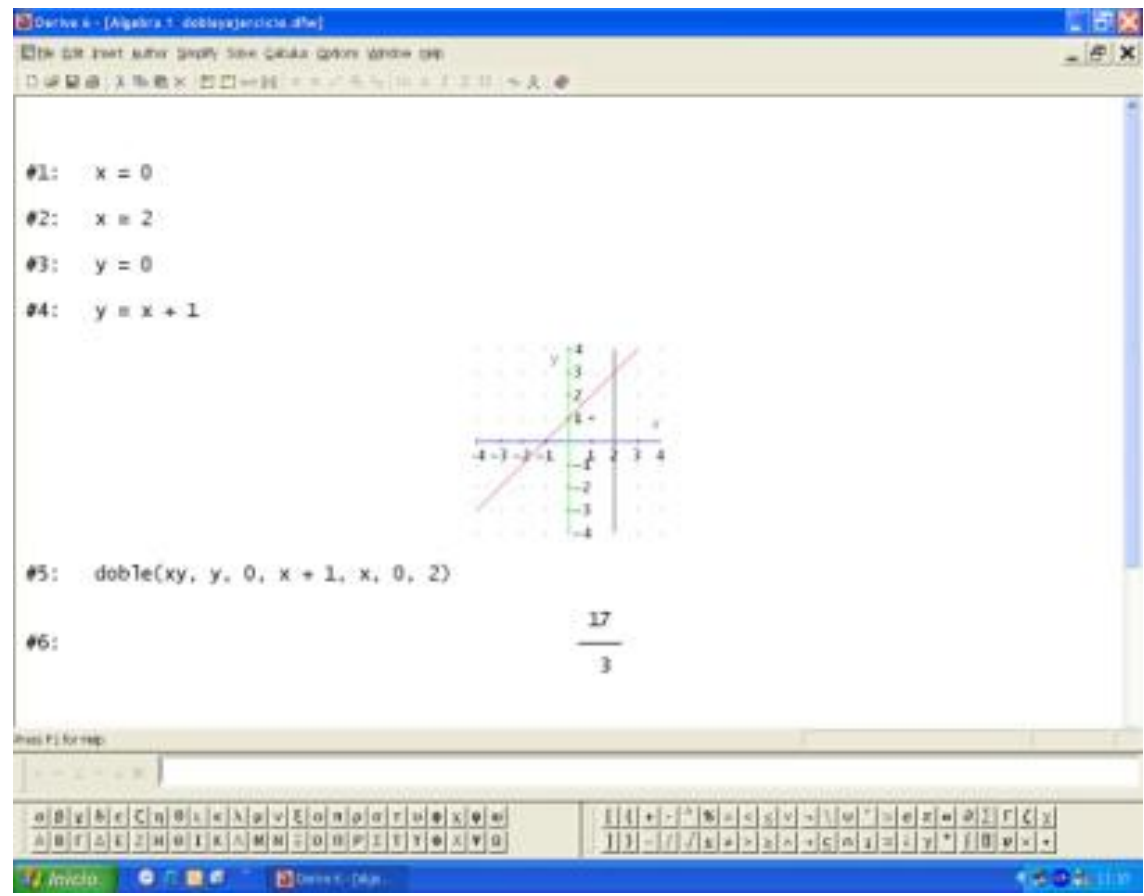




\section{Comando DOBLEPOLAR}

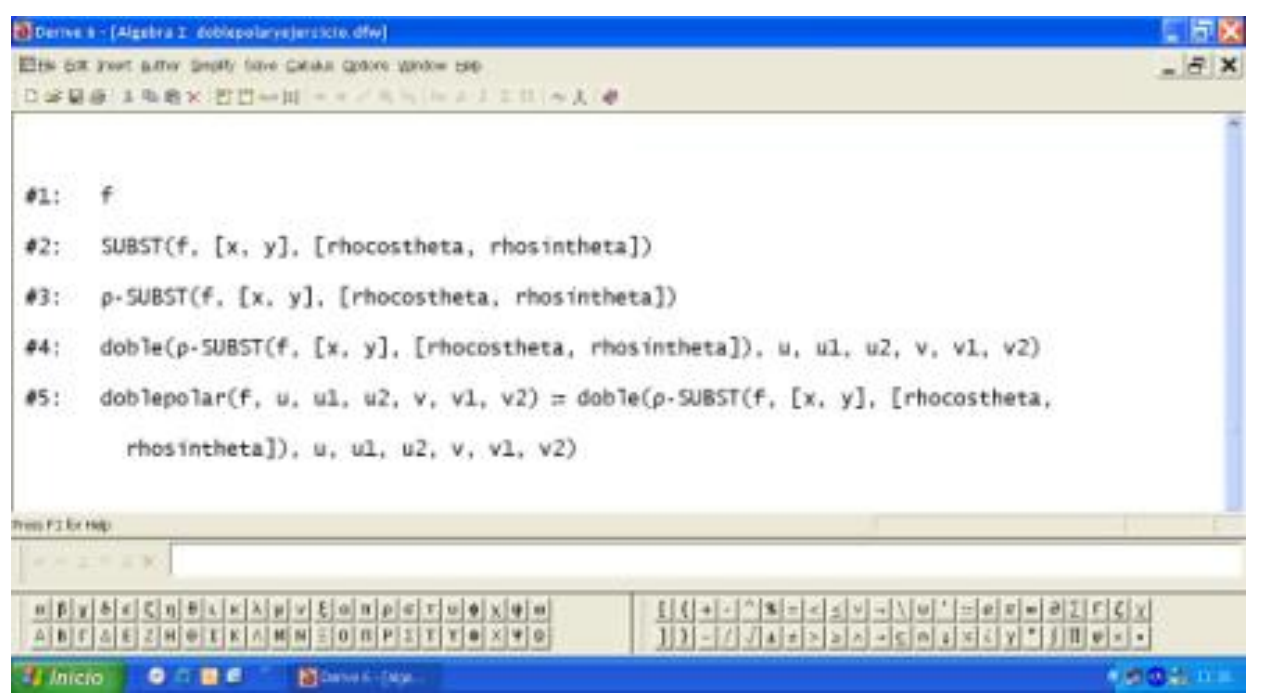

Ilustración 3. Elaboración del comando DOBLEPOLAR [3]

Posteriormente el alumno pasa a resolver el correspondiente ejercicio de comprobación.

- Ejercicio de comprobación: Calcular el área de la circunferencia $x^{2}+y^{2}=4$.

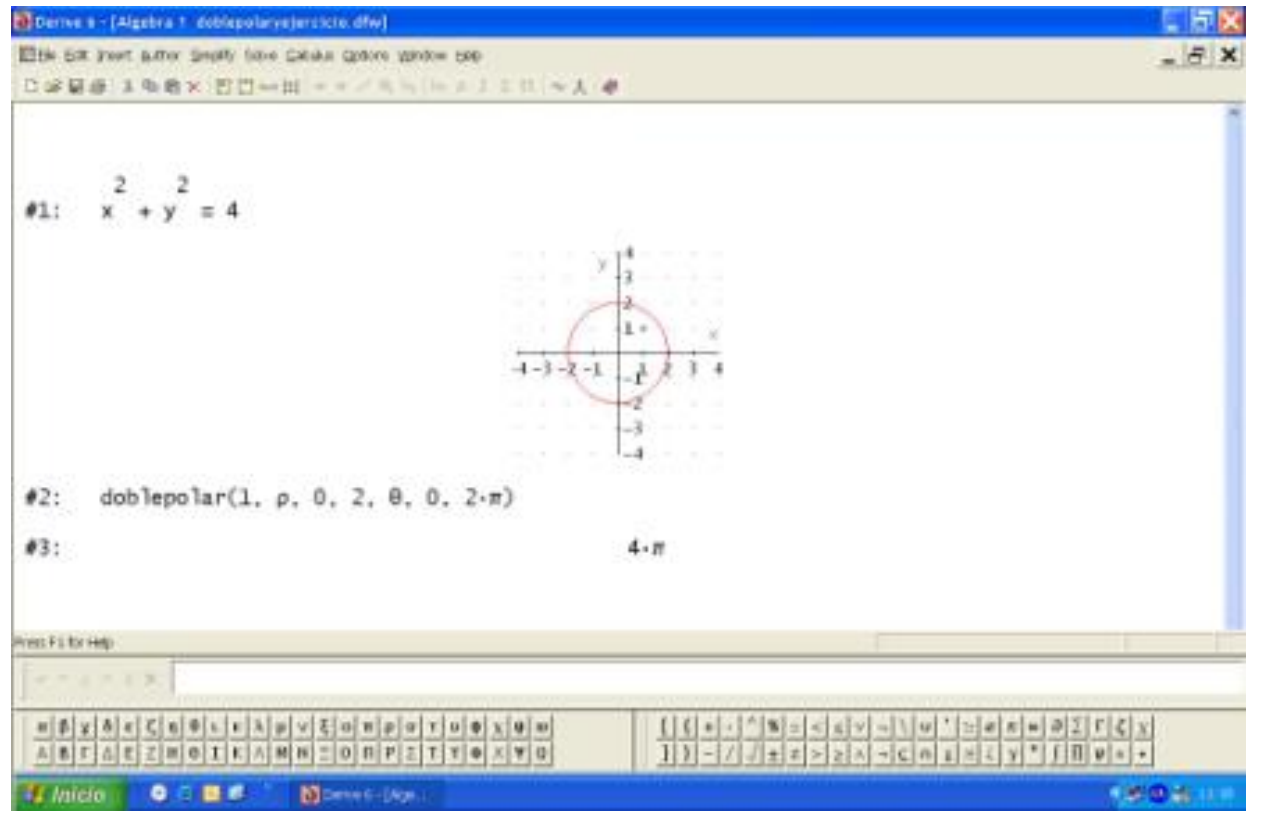

Ilustración 4. Ejercicio de comprobación del comando DOBLEPOLAR [4]

\section{Conclusiones finales.}

Para finalizar, y a modo de resumen, nos gustaría destacar las siguientes conclusiones derivadas de la realización de nuestros estudios:

* Las experiencias acumuladas revelan que los CAS son herramientas informáticas de fácil manejo y útiles para su integración en las clases de Matemáticas de Ingeniería.

* Se deben cambiar los usos tradicionales de los CAS en la enseñanza de las Matemáticas en Ingeniería para maximizar las oportunidades que ofrece esta tecnología. La optimización se debe orientar hacia la mejora de la motivación, la autonomía y el aprendizaje basado en la implicación del alumno en el proceso. 
* Una idea potente es la de combinar los recursos de un CAS con la flexibilidad de un lenguaje de programación.

* Hay indicios razonables de que la realización de comandos con Derive facilita el aprendizaje y mejora la motivación del alumno.

* Aunque sería lo deseable, no es necesario modificar sustancialmente un programa tradicional de una asignatura de Matemáticas en Ingeniería para introducir la elaboración de comandos con Derive por parte de los alumnos.

Además, es importante señalar que se han conseguido alcanzar todos los objetivos comentados en el tercer apartado. Así,

- Comprobar los efectos positivos del desarrollo de una metodología didáctica mixta que incluye la realización de comandos con Derive sobre el aprendizaje.

Tanto las pruebas de evaluación como las encuestas y entrevistas realizadas en las investigaciones que se han llevado paralelamente al desarrollo de las experiencias, avalan la consecución de este objetivo. Conviene destacar la afirmación de los alumnos sobre que la realización de comandos con Derive facilita la asimilación de los contenidos.

- Comprobar la adecuación de la modificación curricular y su compatibilidad con las condiciones usuales del desarrollo de las asignaturas así como con las orientaciones oficiales al respecto.

La propia realización de las prácticas contribuye a la consecución de este objetivo. Dichas prácticas se han incluido en las programaciones oficiales de las asignaturas y han formado parte del proceso de evaluación de las mismas.

- Constatar la incidencia positiva del tratamiento didáctico mencionado sobre los diferentes factores del proceso didáctico, sobre las actitudes, los conocimientos, la participación, las destrezas profesionales y el rendimiento de los alumnos.

Las opiniones de los alumnos vuelven a avalar significativamente la consecución de este objetivo. Dichas opiniones van en el sentido de que Derive facilita y mejora el aprendizaje, la comprensión, la motivación, la comunicación entre alumnos y el rendimiento en exámenes.

- Indagar sobre las posibles incidencias negativas que pueda ocasionar la realización de comandos con Derive sobre el aprendizaje.

Las pruebas de evaluación realizadas en las investigaciones citadas así como las opiniones de los alumnos reafirman la no existencia de variaciones negativas en el aprendizaje por el hecho de la realización de comandos con Derive.

- Fomentar la participación del alumnado en la elaboración y desarrollo de las distintas actividades.

Ya se ha comentado que se ha fomentado en todo momento la participación del alumno en la elaboración y desarrollo de las prácticas que se han realizado. Recordemos que este hecho viene dado porque los alumnos debían desarrollar algunos de los comandos para posteriormente utilizarlos a la hora de resolver los problemas típicos de las asignaturas.

Referencias Bibliográficas.

ABBOUD, M. (2002): Introducing experiments into a first course in calculus. Proceedings of the $2 n d$ International Conference on the Teaching of Mathematics (at the undergraduate level). Hersonissos, Creta (Grecia). 
ALONSO, F.; GARCÍA, A.; GARCÍA, F.; HOYA, S.; RODRÍGUEZ, G. y DE LA VILLA, A. (2001): Some unexpected results using computer algebra systems. The International Journal of Computer Algebra in Mathematics Education, 8(3), 239-252.

BOVIO, M. (2002): Suggestions Focusing Derive at Upper Grades. Pt.3. Examples of Software Errors. L'Insegnamento della Matematica e delle Science Intégrate, 25(2), 111-128.

BÖHM, J. (2002): Programming in DERIVE 5 - introductory example(s). Proceedings of the Vienna International Symposium on Integrating Technology into Mathematics Education (VisitMe). Viena (Austria).

CAMACHO, M. y DEPOOL, R. (2002): Students' attitudes towards Mathematics and computers when using DERIVE in the learning of calculus concepts. International Journal of Computer Algebra in Mathematics Education, 9(4), 259-283.

DUBINSKY, E. (1994): Pedagogical Change in Undergraduate Mathematics Education. MAA Notes, 35, 114-119.

DUBINSKY, E. (1995): ISETL: A Programming Language for Learning Mathematics. Communications on Pure and Applied Mathematics, 48, 1-25.

DUBINSKY, E. (1998): Writing Programs to Learn Mathematics.

http://trident.mcs.kent.edu/ edd/publications.html.

DUBINSKY, E. y NOSS, R. (1996): Some kinds of computers for some kinds of Mathematical learning. Mathematical Intelligencer, 18(1), 17-20.

GALÁN, J.L. (2003): Integrales Múltiples con DERIVE. Un estudio de innovación curricular en primer curso de Ingeniería Técnica de Telecomunicación. Tesis doctoral, Universidad de Málaga, España.

GALÁN, J.L.; GALÁN, M.A.; PADILLA, Y. y RODRÍGUEZ, P. (2002a): Are computers under-used in Mathematical teaching for engineers? Educational technology. Serie Sociedad de la información $\mathrm{n}^{\circ}$ 9, 220-225.

GALÁN, J.L.; GALÁN, M.A.; PADILLA, Y. y RODRÍGUEZ, P. (2002b): Use of the computer in Mathematic teaching for engineers. A powerful calculator? Proceedings of the 2 nd International Conference on the Teaching of Mathematics (at the undergraduate level). Hersonissos, Creta (Grecia).

GARCÍA, A.; GARCÍA, F.; HOYA, S.; RODRÍGUEZ, G. y DE LA VILLA, A. (2002): Differential calculus of several variables with Mathematica or Maple. Proceedings of the 2nd International Conference on the Teaching of Mathematics (at the undergraduate level). Hersonissos, Creta (Grecia).

HECTOR, J. (2002): Problem Solving, Programming and Pedagogy. Proceedings of the Vienna International Symposium on Integrating Technology into Mathematics Education (VisitMe). Viena (Austria).

HOYA, S.; MARTÍN, A.; RODRÍGUEZ, G. y VISUS, I. (2002): The use of symbolic calculus software in the teaching of Mathematics at Engineering Schools. Proceedings of the International Conference on ICT's in Education. Badajoz (España).

MAJEWSKI, M. (2000): Non trivial applications of Maple in teaching Mathematics. Proceedings of the International Conference on Technology in Mathematics Education (ICTME 2000). Beirut (Líbano).

MONAGAN, M. (1994): Worksheets: can we teach mathematical algorithms with them? Maple V: 
Mathematics and its application. Nueva York.

MORPHETT, B. (1997): Using a Computer Algebra System as an aid to problem solving in the secondary school. Proceedings of the 16th Biennial Conference of the Australian Association of Mathematics Teachers. Melbourne (Australia).

NAVA, J. (1998): Experiencia en el uso de programas computacionales para enseñar Matemáticas en Ingeniería en la UNITEC.

http://dcb.fi-c.unam.mx/foro/memorias/dieciocho.pdf.

NEUPER, W. (2001): What teachers can request from CAS-designers. Proceedings of the 5th International Conference on Technology in Mathematics Teaching (ICTMT 5). Klagenfurt (Austria).

ORTEGA, P. (2002): La enseñanza del Álgebra Lineal mediante sistemas informáticos de cálculo algebraico. Tesis doctoral. Universidad Complutense de Madrid, España.

PADILLA, Y. (2003). Integrales de Línea con DERIVE. Un estudio de innovación curricular en primer curso de Ingeniería Técnica de Telecomunicación. Tesis doctoral, Universidad de Málaga, España.

RODRÍGUEZ, P. (2004). Derivación e Integración de Funciones de Variable Compleja con DERIVE. Un estudio de innovación curricular en segundo curso de Ingeniería Técnica de Telecomunicación. Tesis doctoral, Universidad de Málaga, España

STRICKLAND, P. (1999): A Computer Algebra System for improving student's manipulation skills in Algebra. The International Journal of Computer Algebra in Mathematics Education, 6(1), 17-24.

WESTERMANN, T. (2000): Teaching Mathematics using a Computer Algebra. The International Journal of Computer Algebra in Mathematics Education, 7(4), 277-293.

\section{Notas:}

[1] En la expresión \#1 se introduce una función genérica f. En la expresión \#2 se calcula la integral de la función f respecto a una variable genérica u con límites de integración genéricos u1 y u2. En la expresión \#3 se calcula la integral de la expresión \#2 respecto a una nueva variable genérica $\mathrm{v}$ con límites de integración genéricos v1 y v2. Finalmente, en la expresión \#4, se crea el comando DOBLE con sus parámetros y el valor calculado en la expresión \#3. Resaltar la importancia de utilizar tanto variables como límites de integración genéricos para permitir el cálculo de integrales dobles sin tener prefijado el orden de integración.

[2] En las expresiones de la \#1 a la \#4 se introducen las ecuaciones que limitan la región de integración para dibujarla. Después de hacer la representación gráfica, los límites de integración son fácilmente deducibles. En la expresión \#5 se usa el comando DOBLE para calcular la integral doble pedida. En la expresión \#6 se obtiene el resultado del ejercicio.

[3] En la expresión \#1 se introduce una función genérica f. En la expresión \#2 se realiza el cambio a coordenadas polares, es decir, se sustituyen las variables x e y por sus nuevas expresiones. En la expresión \#3 se multiplica la expresión \#2 por el jacobiano de la transformación. En la expresión \#4 se construye la integral utilizando el comando DOBLE antes elaborado. Por último, en la expresión \#5, se construye el comando DOBLEPOLAR con sus argumentos. De nuevo es importante resaltar la importancia de utilizar tanto variables como límites de integración genéricos para permitir el cálculo de integrales dobles sin tener prefijado el orden de integración. Además, a la hora de elaborar comandos se pueden utilizar los que ya están creados previamente, simplificando considerablemente el proceso de construcción. 
[4] En la expresión \#1 se introduce la ecuación que limita la región de integración para representarla. Después de hacer la representación gráfica, los límites de integración son fácilmente deducibles. En la expresión \#2 se usa el comando DOBLEPOLAR para calcular la integral doble pedida. En la expresión \#3 se obtiene el resultado del ejercicio

(C) Ediciones Universidad de Salamanca 